\title{
Parametric cascade downconverter for intense ultrafast mid-infrared generation beyond the Manley-Rowe limit
}

\author{
James M. Fraser and Cathie Ventalon
}

\begin{abstract}
We propose a scheme to generate intense, ultrafast mid-infrared pulses with conversion efficiencies exceeding the upper bound for single-stage difference-frequency mixing as predicted by the Manley-Rowe relations. Finite-element fast Fourier transform simulations of the mixing process show that the parametric cascade downconverter generates 1.7 times more photons (at $10 \mu \mathrm{m}$ ) than in the initial pump pulse (center wavelength of $1.48 \mu \mathrm{m}$, duration of $130 \mathrm{fs}$, and pulse energy of $50 \mu \mathrm{J}$ ), with negligible pulse spatial and temporal distortion. (C) 2006 Optical Society of America
\end{abstract}

OCIS codes: $190.2620,190.4970,320.7160,320.7110$.

Intense and ultrafast optical pulses (durations typically $100 \mathrm{fs}$ or less) are a powerful tool in the fields of nonlinear spectroscopy and ultrafast dynamics. Pulses centered at wavelengths in the mid-infrared (MIR) are well suited to the resonant study of many systems from intersubband transitions in quantum wells to vibrational transitions in molecules. Peak intensities exceeding $\mathrm{TW} / \mathrm{cm}^{2}$ allow strong field interaction, providing novel ways of controlling a system and studying its nonequilibrium properties. ${ }^{1,2}$ Research in this field has been impeded due to a lack of optical sources that directly produce ultrafast intense pulses at long wavelengths. One technique that has provided important results is MIR generation through nonlinear difference-frequency mixing (DFM) starting from intense ultrafast pulses in the near infrared $(800 \mathrm{~nm})$. Recent results that produce intense pulses at kilohertz repetition rates have demonstrated wavelength

J. M. Fraser (fraser@physics.queensu.ca) is with the Department of Physics, Engineering Physics, and Astronomy, Queen's University, Kingston, Ontario K7L 3N6, Canada. When this research was performed, C. Ventalon was with the Laboratoire d'Optique et Biosciences, Centre National de la Recherche Scientifique, Unité Mixte de Recherche 7645, Institut National de la Santé et de la Recherche Médicale U451, Ecole Polytechnique, 91128 Palaiseau Cedex, France. She is now with the Department of Biomedical Engineering, Boston University, 44 Cummington Street, Boston, Massachusetts 02215.

Received 6 September 2005; revised 23 December 2005; accepted 6 January 2006; posted 3 March 2006 (Doc. ID 64595).

0003-6935/06/174109-05\$15.00/0

(C) 2006 Optical Society of America tuning up to $20 \mu \mathrm{m}$, pulse durations around $100 \mathrm{fs}$, and pulse energies of more than $2 \mu \mathrm{J}$ at $10 \mu \mathrm{m} \cdot{ }^{3-7}$

Improving DFM MIR power conversion efficiency is constrained by the basic limitation quantified by the Manley-Rowe relations, ${ }^{8}$ which show that the number of photons output at MIR wavelengths cannot exceed the number of pump photons input; the uppermost quantum efficiency (QE) of the DFM process is $100 \% .{ }^{9}$ The beams involved in DFM are classified pump, signal, and MIR in order of increasing wavelength. If we define DFM power conversion efficiency (PE) as MIR output power divided by input pump power, $\mathrm{PE}$ will always be lower than $\mathrm{QE}$ by

$$
\mathrm{PE}=\mathrm{QE} \frac{\lambda_{\mathrm{PU}}}{\lambda_{\mathrm{MIR}}}
$$

where $\lambda_{\mathrm{PU}}$ and $\lambda_{\mathrm{MIR}}$ are the wavelengths of the pump and MIR beams, respectively. For example, the upper limit on $10 \mu \mathrm{m}$ generation from a $1480 \mathrm{~nm}$ pump beam is $14.8 \%$. In practice, even this limit cannot be reached since $100 \% \mathrm{QE}$ cannot be achieved. A simple one-dimensional solution to Maxwell's equations shows that pump depletion is followed by sumfrequency mixing (SFM) in which energy transfers back to the pump beam. ${ }^{8}$ In the full threedimensional case, the flow of energy is more complicated since different spatial and spectral sections of the pulse have different intensities and thus experience conversion at different rates: one part of the pulse could experience DFM while another is undergoing SFM. No single crystal length would result in 
$100 \%$ conversion. In most cases it is not desirable to approach high conversion efficiency due to the onset of severe pulse degradation. The high conversion is accompanied by pulse reshaping in the temporal, spectral, and spatial domains, making the resulting MIR pulses far from Gaussian in spectrum and shape and difficult to use.

Overcoming the Manley-Rowe limitation requires extending the mixing scheme to multiple stages. Previous research with long duration pulses (100 ps or more) has made use of two stages in an intracavity geometry to achieve greater than $100 \%$ photon conversion. ${ }^{10}$ Tandem optical parametric oscillators have also been demonstrated for high-repetition-rate femtosecond pulses, including work by Tillman et al. to generate wavelengths of up to $4.2 \mu \mathrm{m} .{ }^{11}$ Standard ultrafast microjoule sources have lower repetition rates than what is practical for optical parametric oscillator operation. Here, we propose a technique to produce high-energy MIR pulses through extracavity mixing in multiple stages that exceed the upper limit predicted by the Manley-Rowe relations for a single DFM stage. By using a series of standard nonlinear crystals, we exploit both the generated MIR and the amplified signal beam, which typically are discarded after the DFM stage. The signal beam is particularly useful since it can have more energy than the original pump beam and its wavelength is closer to the MIR wavelength, thus improving the ratio of $\mathrm{PE}$ to $\mathrm{QE}$ [Eq. (1)] and typically reducing group-velocity mismatch. Each subsequent DFM stage uses the amplified signal from the previous stage as the pump to amplify the MIR beam. Note that the proposed process is analogous to the quantum cascade laser, ${ }^{12}$ in which intersubband electrons are reused at each cascade stage to amplify the coherent terahertz beam. The proposed device can be thought of as a parametric cascade downconverter (PCD), in which each cascade stage (DFM crystal) results in the generation of a lower-photon-energy beam (to be used later) and amplification of the coherent MIR beam. We show that the power conversion efficiency of a practical PCD exceeds the theoretical upper bound of a single stage. In addition, the design can make use of multiple passes through the same DFM stage to reduce cost and complexity. Straightforward implementation is possible only since MIR DFM generation happens over a wavelength range in which the crystals can compensate for group velocity walk-off.

Generation of intense MIR through DFM is plagued by many technical difficulties. An appropriate DFM crystal must have a high nonlinear coefficient, high damage threshold, refractive indices that allow phase matching over the desired tuning range, negligible linear absorption of all beams, negligible two-photon absorption of both pump and signal beams, and sufficiently low group-velocity mismatch and spatial walk-off to allow useful interaction lengths. Reviews comparing the properties of different crystals detail advantages and disadvantages of different systems, $, 4,5$ and new crystals continue to be developed and optimized. Once appropriate phase-

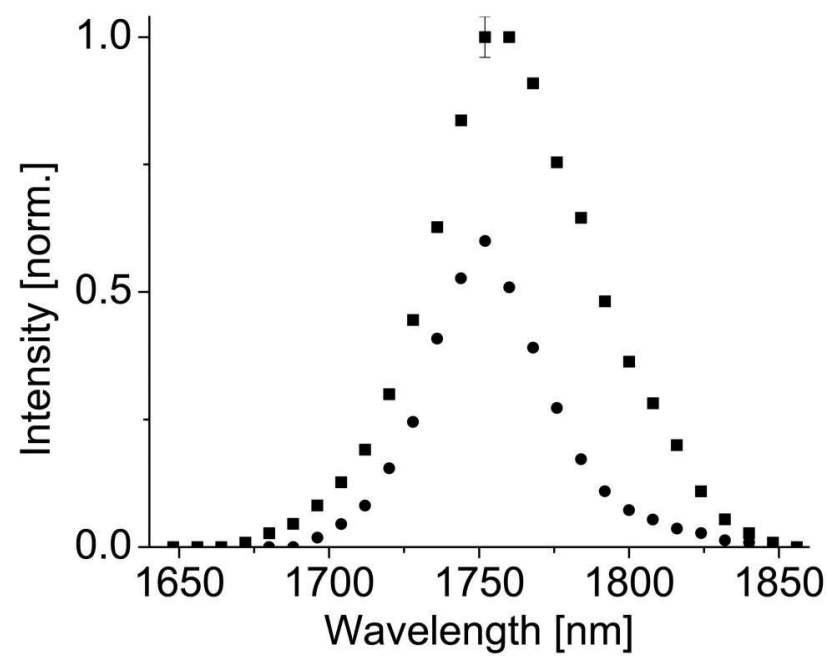

Fig. 1. Signal beam amplification: signal spectrum with (squares) and without (dots) pump present in $1.0 \mathrm{~mm}$ GaSe, measured after the DFM crystal. Data recorded by use of the source described in Ref. 6.

matching conditions are met, the key requirement in crystal selection is a transparency range starting below the MIR photon energy and exceeding twice the photon energy of the pump beam (to avoid two-photon absorption). An assortment of commercially available crystals, such as GaSe, $\mathrm{AgGaS}_{2}$, and $\mathrm{AgGaSe}_{2}$, meet this requirement for pump wavelengths longer than 1000-1420 nm. An optical parametric amplifier (OPA) pumped by a regenerative amplified titaniumdoped sapphire oscillator produces beams in this wavelength range. It is particularly well suited to DFM since it outputs two synchronous pulses separated in frequency by a controllable amount ( 0 to $>170 \mathrm{THz})^{13}$; thus DFM results in an easily tunable MIR beam over a very large wavelength range limited only by nonlinear crystal properties. OPA pulse energies routinely exceed $100 \mu \mathrm{J}$ with 100 fs duration, ${ }^{13}$ and subsequent DFM can result in very high quantum conversion efficiency into MIR. For example, Rotermund and co-workers reported internal quantum efficiencies from $30 \%$ to $80 \%$ over the MIR wavelength range of $4-12 \mu \mathrm{m}$ through DFM in $\mathrm{AgGaS}_{2}$ and $\mathrm{HgGa}_{2} \mathrm{~S}_{4} \cdot{ }^{3}$ The MIR generation is accompanied by large signal power amplification that is often not documented. Figure 1, recorded using the source described previously, ${ }^{6}$ provides a typical result for the nonlinear crystal GaSe. The spectrum of the signal beam after passing through the $1.0 \mathrm{~mm}$ GaSe crystal (type II phase matching) is shown with and without the pump beam (wavelength of $1.49 \mu \mathrm{m}$, pulse energy of $69 \mu \mathrm{J}$, pulse duration of $130 \mathrm{fs}$, and spot size of $1.5 \mathrm{~mm}$ ) present in the crystal. Average power amplification is $71 \%$. In most MIR generation schemes, the amplified signal beam is discarded after the DFM stage even though it often contains more power than the initial pump beam. The PCD exploits this beam and subsequent signal beams to their full advantage to improve MIR power.

Figure 2 outlines the PCD in its most general form. 


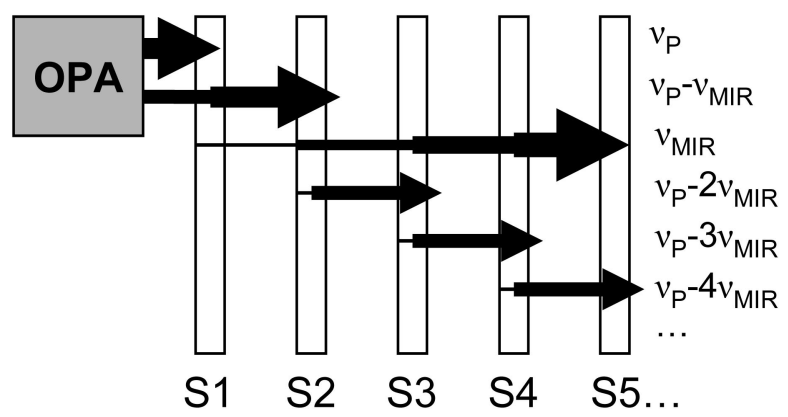

Fig. 2. General parametric cascade downconverter. $\mathrm{S} 1, \mathrm{~S} 2, \ldots$ are DFM crystals. Beams are referenced on the right-hand side and are offset vertically in the figure for purposes of clarity. The frequencies of original pump $\left(v_{P}\right)$, generated MIR $\left(v_{\mathrm{MIR}}\right)$, and intermediate beams are indicated.

The beams are labeled according to their frequencies, with $v_{P}$ and $v_{P}-v_{\mathrm{MIR}}$ being the frequencies of the initial pump and signal beams from the OPA and $v_{\mathrm{MIR}}$ being the frequency of the MIR beam. Each stage exploits the signal beam of the previous stage to amplify the MIR beam, so that the pump frequency decreases by the MIR frequency in each stage. There is a finite limit to the number of stages $\left(v_{P} \operatorname{div} v_{\mathrm{MIR}}\right)$ since only positive frequencies can be considered. Care must be taken at each stage to ensure proper phase matching and temporal and spatial overlap of the pulses. This will be considered in detail below. It is interesting to calculate the power conversion efficiency possible for the PCD in a specific case: pump, signal, and MIR beams at wavelengths of 1.48, 1.74, and $10 \mu \mathrm{m}$, respectively. For a single DFM stage with $f$ quantum conversion efficiency, the power conversion efficiency is $0.148 f$. The corresponding PCD would have six stages and, with a $\mathrm{QE}$ at every stage of $f$, the ideal overall $\mathrm{PE}$ is

$$
\begin{aligned}
\mathrm{PE}_{\mathrm{PCD}}= & \frac{\lambda_{P}}{\lambda_{\mathrm{MIR}}}\left[f+(1+f) f+(1+f) f^{2}+(1+f) f^{3}\right. \\
& \left.+(1+f) f^{4}+(1+f) f^{5}\right] .
\end{aligned}
$$

Here we have assumed that the initial signal beam has the same number of photons as the initial pump beam, as expected from the Manley-Rowe relations that also govern the DFM process in the OPA. The ratio of PE for the PCD compared to a single DFM stage can be written as

$$
\frac{\mathrm{PE}_{\mathrm{PCD}}}{\mathrm{PE}_{\mathrm{SS}}}=\frac{2-f^{5}(1+f)}{1-f} .
$$

Equation (3) and similar expressions for 5 and $15 \mu \mathrm{m}$ generation are shown in Fig. 3. Even for low quantum efficiencies, the PCD provides more than twice the power output from a single DFM stage. As expected, the improvements in MIR increase as a function of MIR wavelength but with the added complexity of additional cascade stages. It is interesting to note the

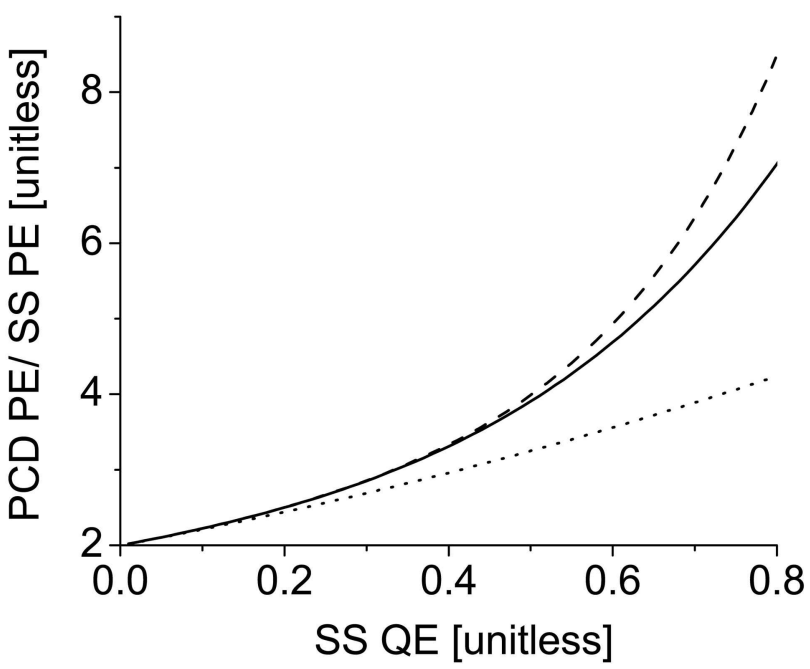

Fig. 3. Ratio of PCD to single-stage (SS) DFM power efficiency as a function of quantum efficiency for three different MIR wavelengths: $5 \mu \mathrm{m}$ (dotted curve), $10 \mu \mathrm{m}$ (solid curve), and $15 \mu \mathrm{m}$ (dashed curve).

required value of $\mathrm{QE}$ for MIR output power to match the pump input power. For $10 \mu \mathrm{m}$ generation, this occurs when $f=0.85$, a value that is likely difficult to attain in practice without serious pulse reshaping. A more realizable benchmark is the $\mathrm{QE}$ value required for the PCD to match the output of a single-stage DFM with $100 \%$ QE. This corresponds to the upper limit predicted by the Manley-Rowe relations but requires a quantum conversion efficiency of only 0.33 in the PCD (for $10 \mu \mathrm{m}$ generation).

Although the above analysis suggests that MIR output can exceed the Manley-Rowe upper limit, it assumes that a constant $\mathrm{QE}(f)$ can be achieved at every stage. We require a more detailed analysis including effects from group-velocity walk-off, spatial walk-off, reflection losses, chirp, and phase matching. The key problem in PCD design is maintaining phase matching and overlap between all relevant pulses at every cascade stage. The feasibility of a sequence of nonlinear crystals interspersed with dispersion and polarization control optics is questionable. To reduce complexity, costs, and footprint, we propose a PCD setup that uses multiple passes through a single

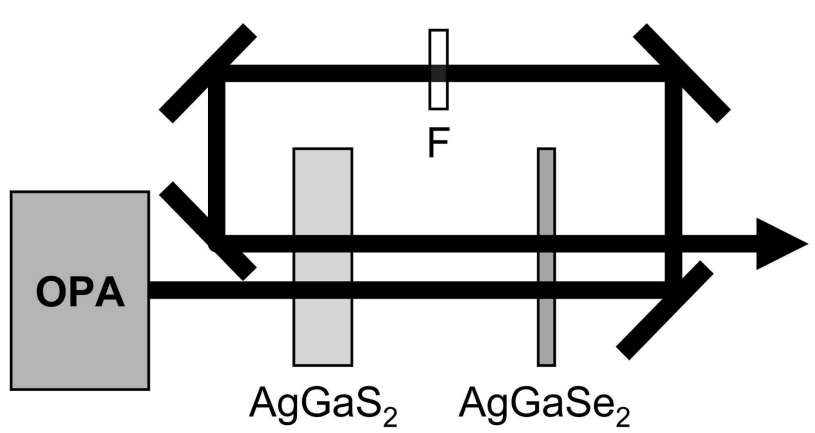

Fig. 4. PCD implementation by use of two nonlinear crystals. F, filter. 
Table 1. Wavelengths and Phase Matching for PCD Implementation

\begin{tabular}{clccccc}
\hline Stage & Crystal & $\begin{array}{c}\text { Pump } \\
(\mu \mathrm{m})\end{array}$ & $\begin{array}{c}\text { Signal } \\
(\mu \mathrm{m})\end{array}$ & $\begin{array}{c}10 \mu \mathrm{m} \\
\text { MIR }\end{array}$ & $\begin{array}{c}\text { PM } \\
\text { Type }\end{array}$ & $\begin{array}{c}\text { PM } \\
\text { Angle }\left(^{\circ}\right)\end{array}$ \\
\hline 1 & $\mathrm{AgGaS}_{2}$ & $1.48(e)$ & $1.74(o)$ & $(o)$ & $\mathrm{I}$ & 33.7 \\
2 & $\mathrm{AgGaSe}_{2}$ & $1.74(e)$ & $2.11(o)$ & $(e)$ & II & 56.5 \\
3 & $\mathrm{AgGaS}_{2}$ & $2.11(e)$ & $2.67(o)$ & $(o)$ & I & 34.4 \\
4 & $\mathrm{AgGaSe}_{2}$ & $2.67(e)$ & $3.64(o)$ & $(e)$ & II & 51.0 \\
\hline
\end{tabular}

pair of crystals (Fig. 4). The crystals, $\mathrm{AgGaS}_{2}$ and $\mathrm{AgGaSe}_{2}$, were chosen for their widespread commercial availability, low reflection loss when properly coated (1\%-3\%), and complementary dispersion properties that provide compensation for temporal pulse walk-off in MIR generation. The latter is a key point: Without the ability to compensate each other for temporal walk-off, the generation process would be much less efficient and the output pulse broadened in time. By using a combination of type I and type II phase matching, no polarization rotation is required between stages. Table 1 lists the wavelengths of each stage along with beam polarizations and phasematching angles. Only a total of four cascade stages are used since the two final stages would provide only a small improvement in MIR power with considerable additional technical difficulty. Table 1 indicates the optimum phase-matching angle for conversion. ${ }^{14,15}$ With the phase-matching schemes employed, the mirrors provide sufficient control to allow $\theta$ to be set to the new phase-matching angle for both stages 3 and 4 with only a small deviation from optimum $\phi$. The sacrifice to amplification is small since the effective nonlinearity is only weakly dependent on $\phi(\cos 2 \phi)$. We also note that amplification sensitivity to the stage 4 phase-matching angle is quite low since this stage has a wide acceptance angle ${ }^{16}\left(20^{\circ}\right.$ for a crystal length of $0.35 \mathrm{~mm}$ ) that is considerably larger than the $5.5^{\circ}$ difference in phase-matching angles from stage 2 .

To provide a quantitative analysis of this implementation, we employed a numerical simulation using a split-step fast Fourier transform analysis for each cascade stage. ${ }^{15}$ Various initial input pulse energies with a common spot size of $1 \mathrm{~mm}$ FWHM and a duration of $130 \mathrm{fs}$ FWHM were tested. The beams output from each DFM crystal are used as the input beams for the subsequent crystal (in terms of pulse energy, spot size, pulse duration, pulse delay, and linear frequency chirp). Spatial walk-off effects are negligible for these spot sizes and thin crystals. We included crystal reflection losses (3\%/surface), crystal linear absorption, and imperfect mirrors (reflectivity of 0.99 ) in the simulation. The crystal lengths are chosen to provide appropriate amplification and new pump generation. The numerical results as a function of input pump energy are shown in Fig. 5 for crystal lengths of 1.0 and $0.35 \mathrm{~mm}$ for the $\mathrm{AgGaS}_{2}$ and $\mathrm{AgGaSe}_{2}$ crystals, respectively. Also shown in Fig. 5 is the upper limit of output power for a single DFM stage, assuming no losses and $100 \%$ quantum conversion efficiency. This limit is exceeded at rela-

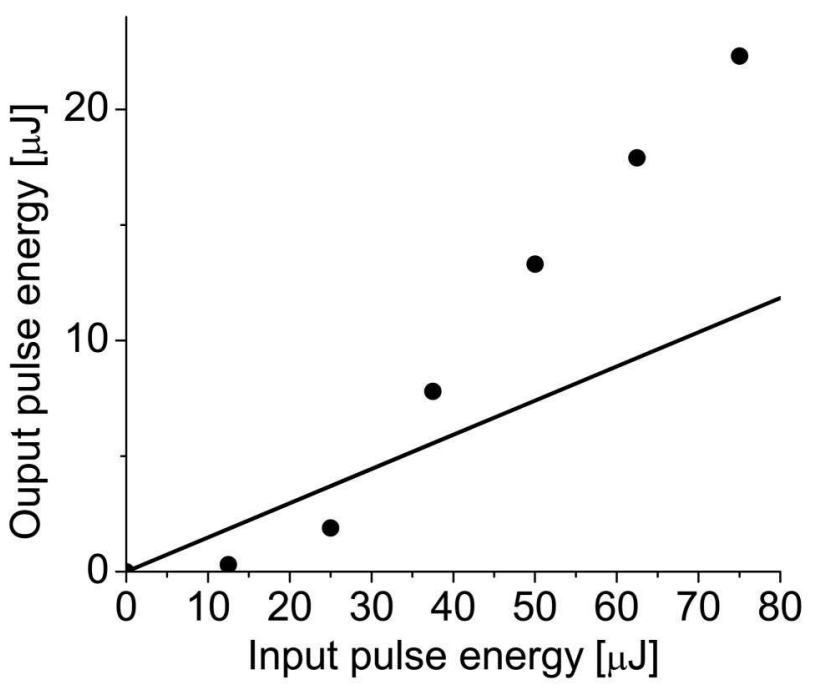

Fig. 5. Numerical simulation results of the PCD $10 \mu \mathrm{m}$ output pulse energy as a function of input $1.48 \mu \mathrm{m}$ pulse energy (dots), compared with the Manley-Rowe upper limit for single-stage DFM (solid line). Simulation parameters are explained in the text.

tively modest input pulse energies. The $13.3 \mu \mathrm{J}$ output pulse energy for $50 \mu \mathrm{J}$ input $(27 \% \mathrm{PE})$ was produced with only a small increase in pulse duration (145 fs FWHM) and is $80 \%$ greater than the upper limit set by Manley-Rowe. For greater input pulse energies, the PE approaches a plateau of $30 \%$ but with the loss of some pulse quality (155 fs pulse duration and $M^{2}$ of 1.1 for $75 \mu \mathrm{J}$ input pulse energy). The spot size of $1.0 \mathrm{~mm}$ was chosen for its ease of use since no focusing or collection optics are required with beams with such long Rayleigh ranges. In practice, the user could employ a telescope before the PCD to adjust the spot size to maximize conversion and achieve better results than those indicated in Fig. 5 for low input pulse energies.

The PCD produces MIR as well as a series of other beams at frequencies separated by the MIR frequency. SFM between these beams must be avoided to avoid depletion of the MIR beam. Phase-matching restrictions clearly prevent most possible cases of unwanted mixing, but there are still two instances that must be considered. Undepleted pump from stage 1 and stage 2 can undergo SFM in stages 3 and 4, respectively. SFM in both cases is guarded against by the introduction of a filter (Fig. 4) that blocks the 1.48 and $1.74 \mu \mathrm{m}$ beams but allows the $2.11 \mu \mathrm{m}$ and MIR beams through with little loss. An antireflectioncoated small bandgap semiconductor, such as InGaAs, would achieve the desired result. An alternative solution would be use of a material that delays the 1.48 and $1.74 \mu \mathrm{m}$ pulses to avoid temporal overlap after a second pass. This might be of interest for studies that require several beams of different frequencies.

We have shown a simple scheme that allows MIR conversion efficiency greater than the upper bound set by the Manley-Rowe relations for single-stage downconversion while avoiding pulse quality degradation. 
In theory, the power output of the PCD can match the input pump power. We have proposed a simple PCD implementation using two passes through two nonlinear crystals for the generation of $10 \mu \mathrm{m}$ light. The crystals serve as sites for nonlinear mixing and also compensate for each other's linear dispersive properties. Numerical simulations indicate that the Manley-Rowe limit for MIR generation can be easily exceeded with negligible reshaping. Advantages of this scheme are even more pronounced for longer MIR generation. For example, the Manley-Rowe relations show that power conversion efficiency in the generation of $1 \mathrm{THz}$ light through optical rectification of $800 \mathrm{~nm}$ light is less than $0.3 \%$. In theory, a properly designed PCD for $1 \mathrm{THz}$ generation could exceed this by 1 or 2 orders of magnitude, but there are serious material issues that would need to be resolved before a terahertz PCD could be achieved.

The experimental results shown in Fig. 1 were recorded at the Laboratoire d'Optique et Biosciences, Ecole Polytechnique, Palaiseau, France. The authors thank Manuel Joffre for fruitful discussions. This research was supported by funding from the National Science and Engineering Research Council.

\section{References and Notes}

1. C. W. Luo, K. Reimann, M. Woerner, T. Elsaesser, R. Hey, and K. H. Ploog, "Phase-resolved nonlinear response of a twodimensional electron gas under femtosecond intersubband excitation," Phys. Rev. Lett. 92, 047402 (2004).

2. C. Ventalon, J. M. Fraser, M. H. Vos, A. Alexandrou, J. L. Martin, and M. Joffre, "Coherent vibrational climbing in carboxyhemoglobin,” Proc. Natl. Acad. Sci. USA 101, 1321613220 (2004).

3. F. Rotermund, V. Petrov, and F. Noack, "Difference-frequency generation of intense femtosecond pulses in the mid-IR
(4-12 $\mu \mathrm{m}$ ) using $\mathrm{HgGa}_{2} \mathrm{~S}_{4}$ and $\mathrm{AgGaS}_{2}$," Opt. Commun. 185, 177-183 (2000).

4. R. A. Kaindl, M. Wurm, K. Reimann, P. Hamm, A. M. Wiener, and M. Woerner, "Generation, shaping, and characterization of intense femtosecond pulses tunable from 3 to $20 \mu \mathrm{m}$," J. Opt. Soc. Am. B 17, 2086-2094 (2000).

5. V. Petrov, F. Rotermund, and F. Noack "Generation of highpower femtosecond light pulses at $1 \mathrm{kHz}$ in the mid-infrared spectral range between 3 and $12 \mu \mathrm{m}$ by second-order nonlinear processes in optical crystals," J. Opt. A Pure Appl. Opt. 3, R1-R19 (2001).

6. C. Ventalon, J. M. Fraser, J. P. Likforman, D. M. Villeneuve, P. B. Corkum, and M. Joffre, "Generation and complete characterization of intense mid-infrared ulrashort pulses," J. Opt. Soc. Am. B 23, 332-340 (2006).

7. Coherent, Inc. technical information on typical OPerA optical parametric amplifier performance running at $1 \mathrm{kHz}$ and pumped with $1 \mathrm{~mJ} /$ pulse at $800 \mathrm{~nm}$.

8. R. W. Boyd, Nonlinear Optics (Academic, 1992).

9. Note that we do not include the input or amplified signal photons in this equation.

10. M. E. Dearborn, K. Koch, G. T. Moore, and J. C. Diels, "Greater than $100 \%$ photon-conversion efficiency from an optical parametric oscillator with intracavity difference-frequency mixing," Opt. Lett. 23, 759-761 (1998).

11. K. A. Tillman, D. T. Reid, D. Artigas, and T. Y. Jiang, "Idlerresonant femtosecond tandem optical parametric oscillator tuning from $2.1 \mu \mathrm{m}$ to $4.2 \mu \mathrm{m}$," J. Opt. Soc. Am. B 21, 15511558 (2004).

12. J. Faist, F. Capasso, D. L. Sivco, C. Sirtori, A. L. Hutchinson, and A. Y. Cho, "Quantum cascade laser," Science 264, 553-556 (1994).

13. G. Cerullo and S. De Silvestri, "Ultrafast optical parametric amplifiers," Rev. Sci. Instrum. 74, 1-18 (2003).

14. E. Takaoka and K. Kato, "Thermo-optic dispersion formula for $\mathrm{AgGaS}_{2}$," Appl. Opt. 38, 4577-4580 (1999).

15. SNLO version 40 nonlinear optics code available from A. V. Smith, Sandia National Laboratories, Albuquerque, N. Mex. 87185-1423.

16. P. N. Butcher and D. Cotter, Elements of Nonlinear Optics (Cambridge U. Press, 1990). 